\title{
Analysis of COVID-19 articles published in dental journals
}

\author{
Parisa Soltani ${ }^{1}$, Kimia Baghaei ${ }^{2}$, Kioumars Tavakoli Tafti ${ }^{2}$, Gianrico Spagnuolo ${ }^{3}$
}

Publisher's Note: MDPI stays neutral with regard to jurisdictional claims in published maps and institutional affiliations.

\section{(c) (P)}

Copyright: (c) 2020 by the authors. Submitted for possible open access publication under the terms and conditions of the Creative Commons Attribution (CC BY) license (http://creativecommons.org/licenses/by/4.0/).

\author{
${ }^{1}$ Department of Oral and Maxillofacial Radiology, Dental Implants Research Center, Dental Research Institute, \\ School of Dentistry, Isfahan University of Medical Sciences, Isfahan, Iran \\ ${ }^{2}$ Dental Students' Research Committee, School of Dentistry, Isfahan University of Medical Sciences, Isfahan, \\ Iran \\ ${ }^{3}$ Department of Neurosciences, Reproductive and Odontostomatological Sciences, University of Naples "Fed- \\ erico II", Naples, Italy
}

\begin{abstract}
Introduction: Coronavirus disease 2019 (COVID-19) pandemic is an ongoing global health crisis with unmatched outcomes and effects. This pandemic has caused an infodemic of article publication in scientific journal. Dental journals have been active in publication of COVID-19 related articles from the beginning of the pandemic. In this report, we present an analysis of the scientific output of dental journals on COVID-19. Methods: Special COVID-19 database of PubMed was searched on December 3, 2020 with applying Dental Journals filter. Data including journal name, country, month of publication, and number of PubMed citations were recorded. Results: The search retrieved a total of 659 articles, of which 28 were excluded as a result of duplicate records, the article being in a language other than English, the article being a correction or unrelated or briefing of other articles. 631 articles were included in the analysis. Oral Diseases has published the most COVID-19 articles (15.1\%), followed by British Journal of Oral and Maxillofacial Surgery $(8.6 \%)$, Journal of Dental Education (7.9\%), British Dental Journal (6.8\%) and Journal of Craniofacial Surgery (6.0\%) (Figure 1). Most of the articles were from researchers from the United States (168), United Kingdom (120), Brazil (83), Italy (64), China (53) and India (35). June 2020 had the highest number of published articles with 107 COVID-19 papers, followed by September 2020 with 92 articles (Figure 2). The number of citations of the published articles ranged from 0 to 406 . Most articles (64.2\%) did not have any citations and only $5.0 \%$ of them had 10 or more citations (Figure 3).Conclusion: The dental community has been active in publication of COVID-19 articles from the beginning of the pandemic. The papers published by dental journals explore issues such as management of clinical practices during the outbreak, infection control in the dental setting, signs and sypmtoms of COVID-19 affecting the oral cavity, and the impact of COVID-19 pandemic on educational and clinical programs.
\end{abstract}

\section{Figures:}



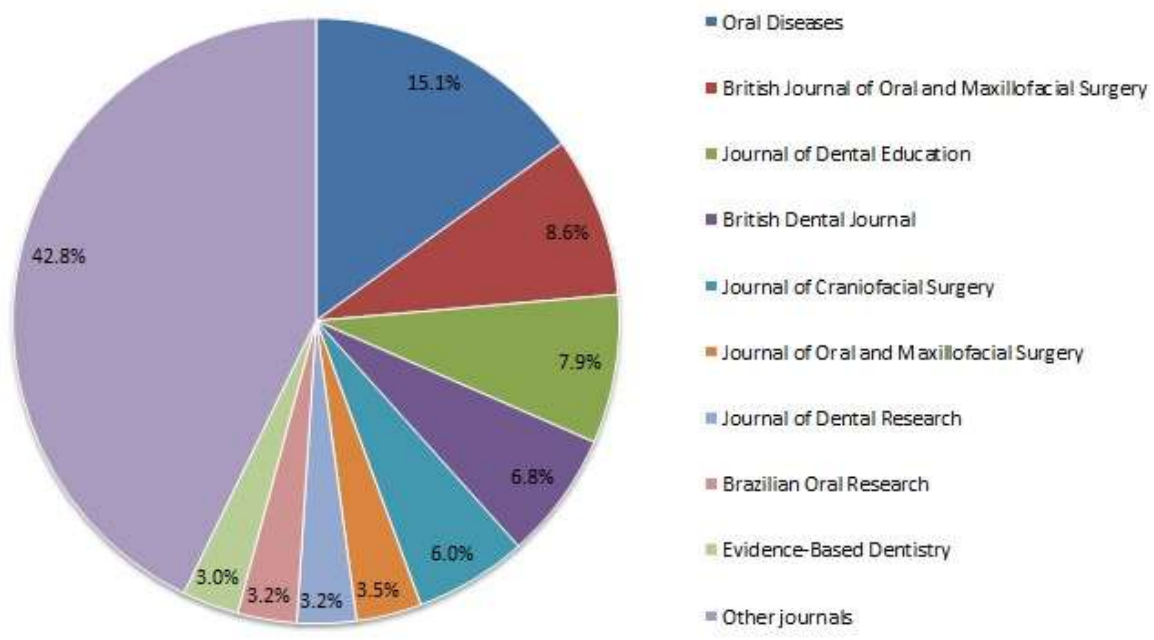

Figure 1. - Journals with the highest number of COVID-19 articles.

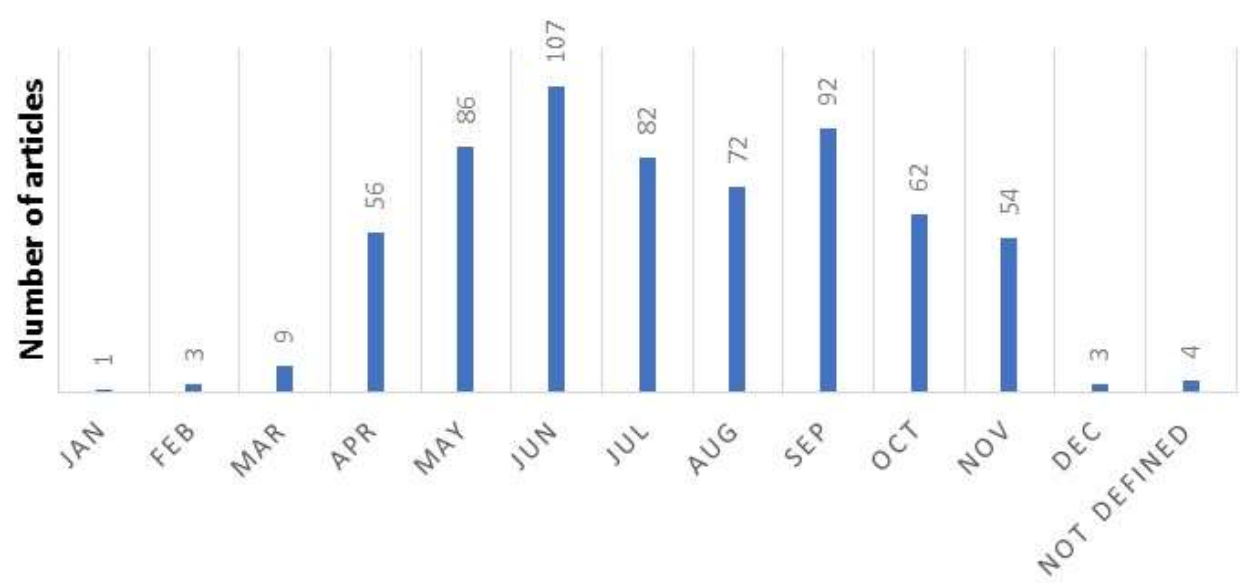

Month of publication

Figure 2. - Frequency of articles published in different months of 2020. 


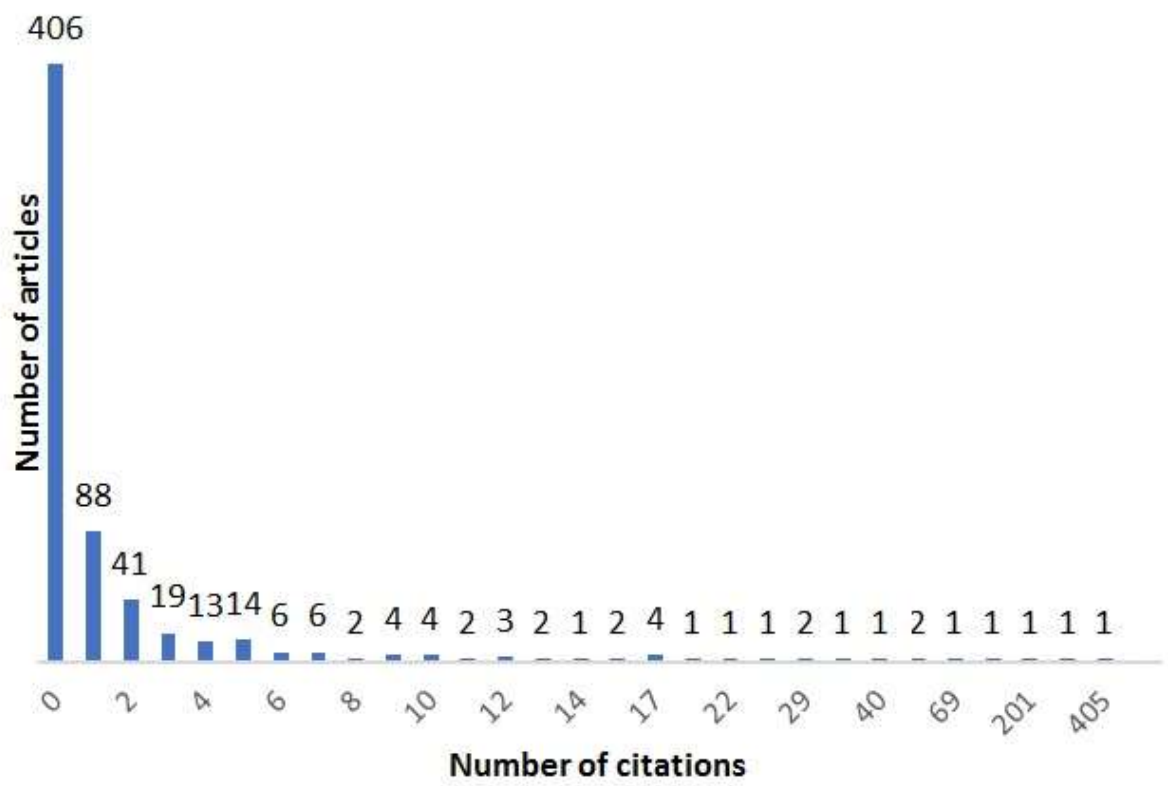

Figure 3. - Frequency of PubMed citations. 